\title{
Hyperfine structure for neutral manganese lines of astrophysical interest
}

\author{
P.-H. Lefèbvre ${ }^{1}$, H.-P. Garnir ${ }^{1}$, and E. Biémont ${ }^{1,2}$ \\ 1 IPNAS (Bât. B15), Université de Liège, Sart-Tilman, 4000 Liège, Belgium \\ 2 Astrophysique et Spectroscopie, Université de Mons-Hainaut, Rue de la Halle, 15, 7000 Mons, Belgium \\ e-mail: E.Biemont@ulg.ac.be
}

Received 4 March 2003 / Accepted 18 April 2003

\begin{abstract}
The hyperfine structure of 40 levels of neutral manganese has been studied by means of Fourier transform emission spectroscopy. The light source was a home-made $99.5 \%$ pure manganese hollow-cathode lamp. From the analysis of the line profiles observed in the visible and infrared regions, we have been able to deduce new magnetic dipole constants for 18 levels and to revise previous results for 22 additional levels.
\end{abstract}

Key words. atomic data - line: profiles

\section{Introduction}

A detailed consideration of hyperfine structure (HFS) is important for stellar abundance determinations. In particular, it has long been recognized that hyperfine structure of neutral manganese can have large effects on stellar absorption line profiles and that the corresponding abundances can be substantially overestimated if such effects are not adequately taken into account in the calculations. In fact, spurious abundance trends may result from the neglect of HFS, the computed results being overestimated for strong lines. For weak transitions, the effect is less critical because such lines are already unsaturated. A long time ago, Heide (1968) estimated that the variations in the logarithmic solar abundances of the iron-group elements could reach 0.1 to 0.2 dex (i.e. 25 up to $50 \%$ ). From an investigation of Mn I lines in F and G stars, Beynon (1978a,b) showed that correct allowance for hyperfine structure was important for deducing accurate manganese abundances relative to iron. This led Booth \& Blackwell (1983) to reconsider quantitatively the effect of hyperfine structure on the analysis of stellar spectra in terms of elemental abundance determination and Booth et al. (1983) to report on measurements of HFS for 53 lines of Mn I observed with the Oxford furnace. More recently, Nissen et al. (2000) investigated the Mn abundance in disk and metal-rich halo stars suggesting that the fact that the Sc and Mn abundance patterns are rather poorly established could be due to the significant importance of HFS affecting the line profiles. Their analysis was relying however only on three Mn I lines (at $\lambda \lambda$ 6013.5, 6016.6 and 6021.8, respectively) for which data were available from measurements by Booth et al. (1983) and results from Steffen (1985). This work was revised

Send offprint requests to: P.-H. Lefèbvre, e-mail: ph.lefebvre@ulg.ac.be by Prochaska \& McWilliam (2000) who investigated again the impact of HFS splittings on stellar abundances and emphasized the fact that an incorrect hyperfine splitting treatment can lead to spurious abundance trends with metallicity.

In astrophysics, a large number of HFS data are needed (see e.g. Kurucz 1993) for statistically significant samples of lines used for abundance determinations. Ideally these data would be provided by laboratory methods, the computations being difficult and frequently inaccurate for complex situations such as those encountered in the iron group elements (see e.g. Olsson \& Rosén 1982 or Andriessen et al. 1976). The most accurate magnetic dipole or electric quadrupole constants or the most precise profile splittings are provided by rf and laser techniques. In Mn I, such data are available only for a very limited number of levels. They have been obtained by Dembczyński et al. (1979) who investigated the $A$ - and $B$-factors of the $3 d^{6} 4 \mathrm{~s}$ ${ }^{6} \mathrm{D}_{1 / 2-9 / 2}$ levels of ${ }^{55} \mathrm{Mn}$ using a laser-rf double resonance method and by Kronfeldt et al. (1985) who determined the magnetic dipole constant $A$ and the electric quadrupole constant $B$ of the $3 \mathrm{~d}^{5} 4 \mathrm{~s} 4 \mathrm{p} \mathrm{z}{ }^{4} \mathrm{P}_{3 / 2,5 / 2}^{\circ}$ levels by means of laser-atomic-beam-spectroscopy. The previous experimental information available is rather old and has been obtained only for a very limited number of levels. More precisely, measurements are due to White \& Ritschl (1930a,b) (prism spectrograph and Fabry-Perot etalon), Wali-Mohammad \& Sharma (1934) (arc in vacuum), Fisher \& Peck (1939)(hollow cathode and Fabry-Perot interferometer), Murakawa (1955) (hollow cathode discharge and Fabry-Perot etalon) and Davis et al. (1971) (spin-exchange optical pumping). The nuclear magnetic moment of ${ }^{55} \mathrm{Mn}$ has been determined by Murakawa \& Kamei (1953) who obtained the value $Q=0.4 \pm 0.2 \times 10^{-24} \mathrm{~cm}^{2}$, 
this value being subsequently revised by Walther (1962). The need of HFS data in Mn I is so marked that recently Melendez (1999) has deduced hyperfine interaction constants and hyperfine components of Mn I lines in the near-infrared region by fitting the solar photospheric spectrum which, as pointed already by Swensson (1966), shows well marked HFS profiles. Such an approach however has to be considered with care because it could be eventually affected by blending problems perturbing the absorption lines.

In order to provide the required HFS information for many levels for which it is needed, an alternative and versatile method is the Fourier transform spectroscopy (FTS) which is able to provide a large number of data of good accuracy with a reasonable effort. For all these reasons, the Fourier transform emission spectroscopy technique has been used in the present analysis and has been able to provide hyperfine structure information for 40 levels of astrophysical interest.

\section{Experimental setup}

The spectrum of neutral manganese, investigated in the present work, was produced with a hollow cathode discharge developed in the laboratory. The lamp was constructed in aluminium, the cathode being a cylinder of $8 \mathrm{~mm}$ diameter made of pure $(99.5 \%)$ manganese prepared from a powder compressed and heated in order to form a solid hollow tube. The covered spectral region extended from 2000 to $35000 \mathrm{~cm}^{-1}$. We have recorded the spectra with a Bruker IFS120 Fourier transform spectrometer having an entrance iris of $1.3 \mathrm{~mm}$. In the infrared region, we used a $\mathrm{CaF}_{2}$ beamsplitter and the resolution was fixed to $0.03 \mathrm{~cm}^{-1}$. The InSb detector used was cooled down with liquid nitrogen. The hollow cathode was filled with a mixture of $\mathrm{He}(14 \%), \mathrm{Ne}(57 \%)$ and $\mathrm{Ar}(29 \%)$ at a total pressure of 3 mbar and a stabilized DC power supply (FUG MCA-3000) was necessary to set the discharge current at $200 \mathrm{~mA}$. In the region 9000-22000 $\mathrm{cm}^{-1}$, a quartz beamsplitter was considered with a Si diode to register the spectra. For these measurements, every interferogram was recorded and stored on an SCSI hard disk and a phase correction procedure was applied individually before the co-addition process. The advantages of this method consist in a reduction of the acquisition time and in the rejection of the "false" interferograms due to arc discharges in the source. The gas mixture, inside the hollow cathode, was similar to that considered in the case of the infrared spectrum and the total pressure was approximately 2 mbar. The current applied to the lamp was fixed at $900 \mathrm{~mA}$. Finally, for the region 22000 $35000 \mathrm{~cm}^{-1}$, outside the spectral range accessible with our instrument, we used additional FTS spectra obtained at the Solar Observatory on Kitt Peak. This spectrum (990311R0.014) was available on the Kitt Peak server. It was registered with an entrance iris of $8 \mathrm{~mm}$ and a resolution of $0.02 \mathrm{~cm}^{-1}$. The light source was a manganese hollow cathode filled with $\mathrm{Ne}(99.8 \%)$ and a trace of nitrogen $(0.2 \%)$. The lamp was operated with a current of $376 \mathrm{~mA}$ at a pressure of 2.005 mbar. More details can be found on the website: http://diglib.nso. edu.

\section{Hyperfine structure model used}

The interaction between nuclear moments (magnetic dipole moment $\mu_{I}$ and electric quadrupole moment $Q_{I}$ ) and the the field due to electrons considered at the nucleus position leads, for hyperfine levels, to Casimir's formula (Casimir 1963)

$W_{F}=W_{J}+A \frac{C}{2}+B \frac{3 C(C+1)-4 J(J+1) I(I+1)}{8 J(2 J-1) I(2 I-1)}$

with

$$
\begin{aligned}
& C=F(F+1)-I(I+1)-J(J+1) \\
& A=-\mu_{I} \frac{\langle\alpha I J F|H(0)| \alpha I J F\rangle}{I . J}
\end{aligned}
$$

$B=e Q_{I}\left\langle\alpha I J F\left|V_{z z}(0)\right| \alpha I J F\right\rangle$

where $<H(0)>$ is the magnetic field due to electrons considered at the nucleus position, $W_{J}$ is the fine structure level energy, $J$ is the angular electronic moment, $F$ is defined by $\boldsymbol{F}=\boldsymbol{I}+\boldsymbol{J}, I$ being the nuclear spin, $\left\langle V_{z z}(0)\right\rangle$ is the electric field gradient at the nucleus position and, finally, $A$ and $B$ are the magnetic dipole and electric quadrupole constants respectively. If we consider an E1 transition between two hyperfine levels, the line strength can be computed following the HönlKronig rule:

$$
S\left(\alpha I J F ; \alpha^{\prime} I J^{\prime} F^{\prime}\right)=(2 I+1) Q\left(I J F ; I J^{\prime} F^{\prime}\right) S\left(\alpha J ; \alpha^{\prime} J^{\prime}\right)
$$

with

$Q\left(I J F ; I J^{\prime} F^{\prime}\right)=\frac{(2 F+1)\left(2 F^{\prime}+1\right)}{(2 I+1)}\left\{\begin{array}{ccc}J & F & I \\ F^{\prime} & J^{\prime} & 1\end{array}\right\}^{2}$

where $S\left(\alpha J ; \alpha^{\prime} J^{\prime}\right)$ is the line strength between two finestructure levels and $Q\left(I J F ; I J^{\prime} F^{\prime}\right)$ is the relative intensity of the hyperfine components of a fine-structure transition ${ }^{1}$. In the present analysis, a synthetic spectral profile $B_{S}(\sigma)$ has been obtained by the convolution of an ideal profile $B(\sigma)$ and an experimental one ILS (Bell 1972):

$B_{S}(\sigma)=B(\sigma) * I L S$.

This profile was compared to the observed profile. If the spectra are not apodized, the experimental profile can be written as:

$(I L S)_{1}=2 L \frac{\operatorname{sinc}(2 \pi L \sigma)}{2 \pi L \sigma}$

where $L$ is the maximum path difference of the Michelson interferometer.

$(I L S)_{2}=\frac{\pi}{\sigma \Omega} \prod\left(0, \frac{\sigma \Omega}{2 \pi}\right)$

with $\Omega=\frac{\pi h^{2}}{4 F^{2}}, h$ being the spectrometer iris diameter and $F$ the collimator focal length.

$(I L S)=(I L S)_{1} *(I L S)_{2}$.

\footnotetext{
${ }^{1}$ For people not familiar with the 6j-symbols, tabulated components have been published in Cowan (1981).
} 


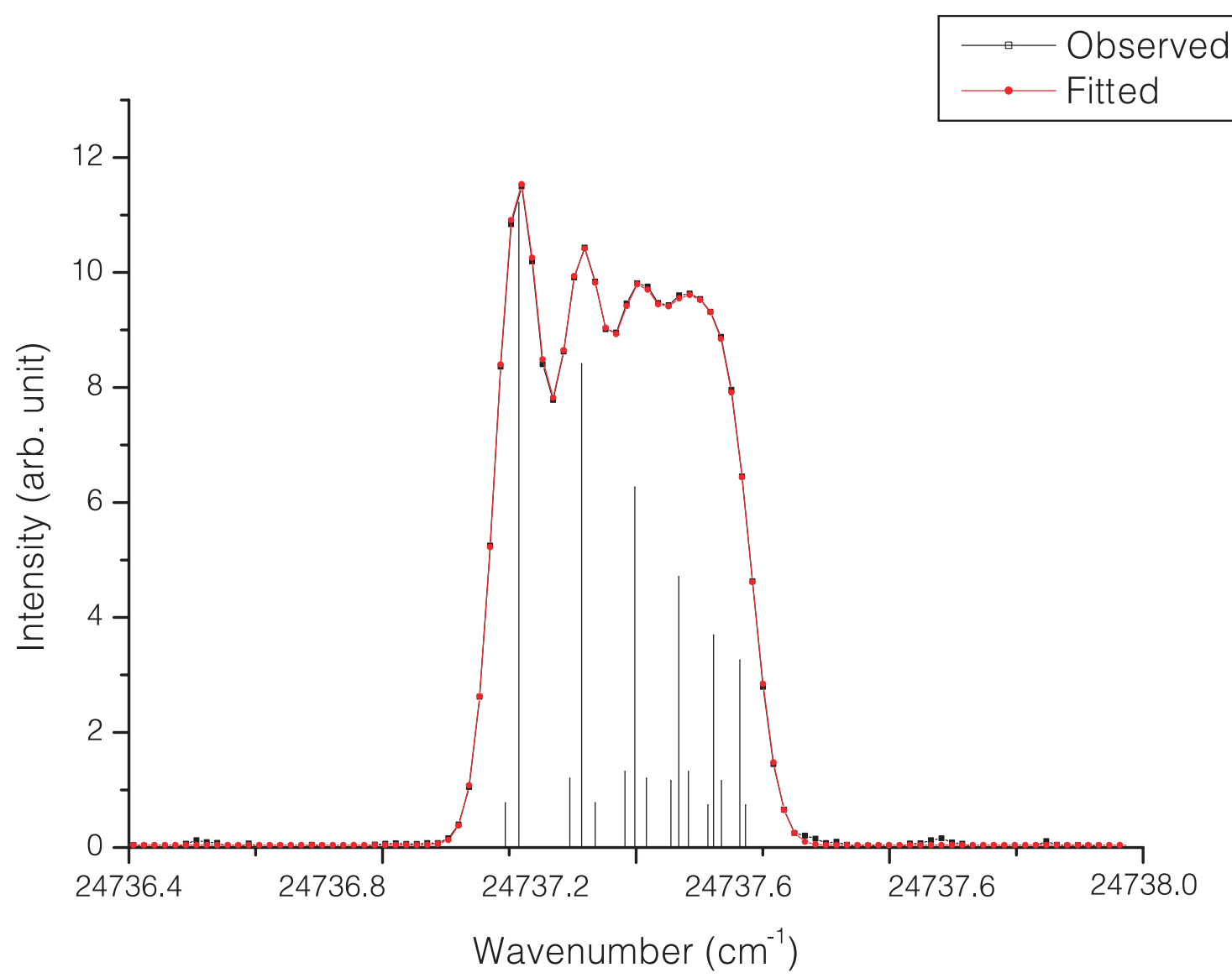

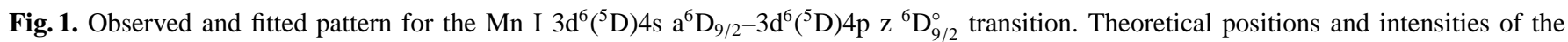
hyperfine structure components are indicated inside of the profile.

The ideal profile used in this model is given by:

$B(\sigma)=k \sum_{F \rightarrow F^{\prime}} Q\left(I J F ; I J^{\prime} F^{\prime}\right) G_{\sigma_{F \rightarrow F^{\prime}, T}}(\sigma)$

where $k$ is a proportionality factor linked to the line strength $S\left(\alpha J ; \alpha^{\prime} J^{\prime}\right)$ and to the atom population in the initial state. The sum is extended to all the hyperfine components $F \rightarrow F^{\prime}$ of a fine structure transition $J \rightarrow J^{\prime} . G_{\sigma_{F \rightarrow F^{\prime}}}(\sigma)$ is the Doppler profile which depends essentially upon the source temperature $T$ and which is centered on $\sigma_{F \rightarrow F^{\prime}}$. The adjustable parameters are the hyperfine constants $A$ and $B$ of the two levels of fine structure considered for the transition, the zero order wavenumber, $\sigma_{0}=W_{J}-W_{J^{\prime}}$, the source temperature $T$ and the coefficient $k$. This method is similar to that used by Palmeri et al. (1995, 1997).

\section{Results and discussion}

From the observation of the Mn I HFS patterns of more than 70 transitions, it was possible to derive the magnetic dipole constants for 40 levels. Among these, the $A$-values have been determined for the first time for 18 levels. The results are reported in Table 1 where they are compared to previous results when available. It should be emphasized that, for all these HFS transitions, at least one of the two magnetic dipole constants (either for the lower or for the upper level of the transition) was known from previous analyses. It was then possible to derive the $A$-value for the other level involved in the transition from a fitting procedure of the calculated profile to the observed pattern according to the procedure described in Sect. 3. The procedure followed here has been used with success previously by Palmeri et al. (1995, 1997), Aboussaid et al. (1996) and Lefèbvre et al. (2002) in the cases of V I, Al II and Sc II. For nearly all the levels, several profiles were used to determine the $A$-values. In all the cases, the observed profiles were nicely reproduced. A comparison between the observed and calculated profiles is illustrated in Fig. 1 in a specific case. The attempt to derive electric quadrupole constants failed, the B-values of the levels considered here being obviously very small. The present results have been compared with previously available theoretical or experimental data for 22 levels (see Table 1). The agreement is good (within 10\%) for 10 levels. Large discrepancies are observed for 12 levels for which only the results of Beynon (1977) are available. This is not unexpected because the uncertainties affecting the results of Beynon (1977) are very large, those values being either obtained from a re-analysis of older data or being theoretical estimates possibly very inaccurate. The new HFS results obtained in the present analysis are expected to be more accurate than the previous results published 
Table 1. New magnetic dipole constants in $\mathrm{Mn} \mathrm{I}\left(\mathrm{A}\right.$, in $\left.10^{-3} \mathrm{~cm}^{-1}\right)$ and comparison with previous results.

\begin{tabular}{|c|c|c|c|c|c|}
\hline \multirow[t]{2}{*}{ Configuration } & \multirow[t]{2}{*}{ Level $^{*}$} & \multirow[t]{2}{*}{$E\left(\mathrm{~cm}^{-1}\right)^{*}$} & \multicolumn{2}{|c|}{$A\left(10^{-3} \mathrm{~cm}^{-1}\right)$} & \multirow[t]{2}{*}{ Ref. } \\
\hline & & & This work & Previous & \\
\hline $3 d^{5} 4 s^{2}$ & $a{ }^{6} S_{5 / 2}$ & 0 & & -2.4157 & Davis et al. $(1971)^{a}$ \\
\hline \multirow[t]{5}{*}{$3 d^{6}\left({ }^{5} D\right) 4 s$} & $a^{6} D_{9 / 2}$ & 17052.29 & & 17.022 & Dembczyński et al. (1979) $b$ \\
\hline & $\mathrm{a}^{6} \mathrm{D}_{7 / 2}$ & 17282.00 & & 15.308 & Dembczyński et al. (1979) ${ }^{b}$ \\
\hline & $a^{6} D_{5 / 2}$ & 17451.52 & & 14.567 & Dembczyński et al. (1979) ${ }^{b}$ \\
\hline & $\mathrm{a}^{6} \mathrm{D}_{3 / 2}$ & 17568.48 & & 15.657 & Dembczyński et al. (1979) ${ }^{b}$ \\
\hline & $a{ }^{6} D_{1 / 2}$ & 17637.15 & & 29.422 & Dembczyński et al. (1979) \\
\hline \multirow[t]{3}{*}{$3 d^{5}\left({ }^{6} S\right) 4 s 4 p$} & $\mathrm{z}^{8} \mathrm{P}_{5 / 2}^{\circ}$ & 18402.46 & & 19.08 & Handrich et al. (1969) ${ }^{c}$ \\
\hline & $\mathrm{z}^{8} \mathrm{P}_{7 / 2}^{\circ}$ & 18531.64 & & 18.23 & Handrich et al. (1969) ${ }^{c}$ \\
\hline & $\mathrm{z}^{8} \mathrm{P}_{9 / 2}^{\circ}$ & 18705.37 & & 14.01 & Handrich et al. (1969) ${ }^{c}$ \\
\hline \multirow[t]{4}{*}{$3 d^{6}\left({ }^{5} D\right) 4 s$} & $\mathrm{a}^{4} \mathrm{D}_{7 / 2}$ & 23296.67 & $-5.5(3)$ & -5.7 & Melendez (1999) ${ }^{d}$ \\
\hline & $a^{4} D_{5 / 2}$ & 23549.20 & $-4.7(2)$ & -4.6 & Melendez (1999) ${ }^{d}$ \\
\hline & $a^{4} D_{3 / 2}$ & 23719.52 & $0.9(4)$ & 0 & Beynon $(1977)^{e}$ \\
\hline & $a{ }^{4} D_{1 / 2}$ & 23818.87 & $50.4(9)$ & 37 & Beynon $(1977)^{e}$ \\
\hline \multirow[t]{3}{*}{$3 d^{5}\left({ }^{6} S\right) 4 s 4 p\left({ }^{3} P^{\circ}\right)$} & $\mathrm{z}^{6} \mathrm{P}_{3 / 2}^{\circ}$ & 24779.32 & & 19.07 & Handrich et al. $(1969)^{c}$ \\
\hline & $\mathrm{z}^{6} \mathrm{P}_{5 / 2}^{\circ}$ & 24788.05 & & 15.591 & Handrich et al. (1969) ${ }^{c}$ \\
\hline & $\mathrm{z}^{6} \mathrm{P}_{7 / 2}^{\circ}$ & 24802.25 & & 14.312 & Handrich et al. (1969) ${ }^{c}$ \\
\hline \multirow[t]{4}{*}{$3 d^{5} 4 s^{2}$} & $\mathrm{a}^{4} \mathrm{G}_{11 / 2}$ & 25265.74 & $13.6(5)$ & & \\
\hline & $a^{4} G_{9 / 2}$ & 25285.43 & $13.7(5)$ & & \\
\hline & $a^{4} G_{7 / 2}$ & 25287.74 & $15.0(5)$ & & \\
\hline & $a{ }^{4} G_{5 / 2}$ & 25281.04 & $20.2(2)$ & & \\
\hline \multirow[t]{3}{*}{$3 d^{5}\left({ }^{6} S\right) 4 s 4 p\left({ }^{3} P^{\circ}\right)$} & $\mathrm{z}^{4} \mathrm{P}_{5 / 2}^{\circ}$ & 31001.15 & & -20.3 & Kronfeldt et al. $(1985)^{f}$ \\
\hline & $\mathrm{z}^{4} \mathrm{P}_{3 / 2}^{\circ}$ & 31076.42 & & -27.4 & Kronfeldt et al. $(1985)^{f}$ \\
\hline & $\mathrm{z}^{4} \mathrm{P}_{1 / 2}^{\circ}$ & 31124.95 & $-73.2(5)$ & -56 & Beynon $(1977)^{e}$ \\
\hline \multirow[t]{3}{*}{$3 d^{5}\left({ }^{6} S\right) 4 s 4 p\left({ }^{1} P^{\circ}\right)$} & $\mathrm{y}^{6} \mathrm{P}_{3 / 2}^{\circ}$ & 35689.98 & & -32.4 & Luc \& Gerstenkorn $(1972)^{g}$ \\
\hline & $\mathrm{y}^{6} \mathrm{P}_{5 / 2}^{\circ}$ & 35725.85 & & -18.0 & Luc \& Gerstenkorn $(1972)^{g}$ \\
\hline & $\mathrm{y}^{6} \mathrm{P}_{7 / 2}^{\circ}$ & 35769.97 & & -13.0 & Luc \& Gerstenkorn $(1972)^{g}$ \\
\hline $3 d^{5}\left({ }^{7} S\right) 5 s$ & $\mathrm{e}^{8} \mathrm{~S}_{7 / 2}$ & 39431.31 & $25.2(5)$ & 24 & Beynon $(1977)^{e}$ \\
\hline $3 d^{5}\left({ }^{7} S\right) 5 s$ & $\mathrm{e}^{6} \mathrm{~S}_{5 / 2}$ & 41403.93 & & (29) & Beynon $(1977)^{e}$ \\
\hline \multirow[t]{5}{*}{$3 d^{6}\left({ }^{5} D\right) 4 p$} & $z^{6} D_{9 / 2}^{\circ}$ & 41789.48 & $3.1(1)$ & 3.5 & Beynon $(1977)^{e}$ \\
\hline & $\mathrm{z}^{6} \mathrm{D}_{7 / 2}^{\circ}$ & 41932.64 & $1.4(2)$ & 2 & Beynon $(1977)^{e}$ \\
\hline & $\mathrm{z}^{6} \mathrm{D}_{5 / 2}^{\circ}$ & 42053.73 & $-8.3(5)$ & 3 & Beynon $(1977)^{e}$ \\
\hline & $\mathrm{z}^{6} \mathrm{D}_{3 / 2}^{\circ}$ & 42143.57 & $-4.7(2)$ & -3.5 & Beynon $(1977)^{e}$ \\
\hline & $z^{6} D_{1 / 2}^{\circ}$ & 42198.56 & $-26.3(4)$ & $(-8)$ & Beynon $(1977)^{e}$ \\
\hline \multirow[t]{5}{*}{$3 d^{6}\left({ }^{5} D\right) 4 p$} & $\mathrm{z}^{6} \mathrm{~F}_{11 / 2}^{\circ}$ & 43314.23 & $6.0(5)$ & & \\
\hline & $\mathrm{z}^{6} \mathrm{~F}_{9 / 2}^{\circ}$ & 43428.58 & $5.2(4)$ & & \\
\hline & $\mathrm{z}^{6} \mathrm{~F}_{7 / 2}^{\circ}$ & 43524.08 & $4.6(3)$ & & \\
\hline & $\mathrm{z}^{6} \mathrm{~F}_{5 / 2}^{\circ}$ & 43595.50 & $5.8(3)$ & & \\
\hline & $\mathrm{z}^{6} \mathrm{~F}_{3 / 2}^{\circ}$ & 43644.45 & $9.1(5)$ & & \\
\hline
\end{tabular}


Table 1. continued.

\begin{tabular}{|c|c|c|c|c|c|}
\hline \multirow[t]{2}{*}{ Configuration } & \multirow[t]{2}{*}{ Level* $^{*}$} & \multirow[t]{2}{*}{$E\left(\mathrm{~cm}^{-1}\right)^{*}$} & \multicolumn{2}{|c|}{$A\left(10^{-3} \mathrm{~cm}^{-1}\right)$} & \multirow[t]{2}{*}{ Ref. } \\
\hline & & & This work & Previous & \\
\hline & $\mathrm{z}^{6} \mathrm{~F}_{1 / 2}^{\circ}$ & 43672.66 & & & \\
\hline \multirow[t]{4}{*}{$3 d^{6}\left({ }^{5} D\right) 4 p$} & $z^{4} F_{9 / 2}^{\circ}$ & 44288.76 & $4.5(5)$ & (5) & Beynon $(1977)^{e}$ \\
\hline & $z^{4} F_{7 / 2}^{\circ}$ & 44523.45 & $6.0(4)$ & 6 & Beynon $(1977)^{e}$ \\
\hline & $\mathrm{z}^{4} \mathrm{~F}_{5 / 2}^{\circ}$ & 44696.29 & $9.9(4)$ & 10 & Beynon $(1977)^{e}$ \\
\hline & $\mathrm{z}^{4} \mathrm{~F}_{3 / 2}^{\circ}$ & 44814.23 & $22.4(9)$ & 18 & Beynon $(1977)^{e}$ \\
\hline \multirow[t]{3}{*}{$3 d^{6}\left({ }^{5} D\right) 4 p$} & $\mathrm{x}^{6} \mathrm{P}_{7 / 2}^{\circ}$ & 44993.92 & $9.1(3)$ & & \\
\hline & $\mathrm{x}^{6} \mathrm{P}_{5 / 2}^{\circ}$ & 45156.11 & $8.7(9)$ & & \\
\hline & $\mathrm{x}^{6} \mathrm{P}_{3 / 2}^{\circ}$ & 45259.17 & $12(2)$ & & \\
\hline \multirow[t]{4}{*}{$3 d^{6}\left({ }^{6} D\right) 4 p$} & $\mathrm{z}^{4} \mathrm{D}_{7 / 2}^{\circ}$ & 45754.27 & & 5 & Beynon $(1977)^{e}$ \\
\hline & $\mathrm{z}^{4} \mathrm{D}_{5 / 2}^{\circ}$ & 45940.93 & $1.9(3)$ & 6 & Beynon $(1977)^{e}$ \\
\hline & $\mathrm{z}^{4} \mathrm{D}_{3 / 2}^{\circ}$ & 46083.89 & $6.1(3)$ & 8 & Beynon $(1977)^{e}$ \\
\hline & $z^{4} D_{1 / 2}^{\circ}$ & 46169.93 & $35.0(5)$ & 19 & Beynon $(1977)^{e}$ \\
\hline \multirow[t]{3}{*}{$3 d^{5} 4 s\left({ }^{7} S\right) 5 p$} & $\mathrm{y}^{8} \mathrm{P}_{5 / 2}^{\circ}$ & 45981.44 & & 27.5 & Melendez (1999) ${ }^{d}$ \\
\hline & $\mathrm{y}^{8} \mathrm{P}_{7 / 2}^{\circ}$ & 46000.77 & & 20.0 & Melendez (1999) ${ }^{d}$ \\
\hline & $\mathrm{y}^{8} \mathrm{P}_{9 / 2}^{\circ}$ & 46026.75 & & 16.5 & Melendez (1999) ${ }^{d}$ \\
\hline \multirow[t]{5}{*}{$3 d^{5} 4 s\left({ }^{7} S\right) 4 d$} & $\mathrm{e}^{8} \mathrm{D}_{3 / 2}$ & 46706.09 & $39(4)$ & & \\
\hline & $e^{8} D_{5 / 2}$ & 46707.03 & $22(2)$ & & \\
\hline & $\mathrm{e}^{8} \mathrm{D}_{7 / 2}$ & 46708.33 & $19(2)$ & & \\
\hline & $\mathrm{e}^{8} \mathrm{D}_{9 / 2}$ & 46710.15 & $15(1)$ & & \\
\hline & $e^{8} D_{11 / 2}$ & 46712.58 & $14.0(8)$ & & \\
\hline \multirow[t]{3}{*}{$3 d^{6}\left({ }^{5} D\right) 4 p$} & $\mathrm{y}^{4} \mathrm{P}_{5 / 2}^{\circ}$ & 46901.13 & $-1.8(4)$ & (3) & Beynon $(1977)^{e}$ \\
\hline & $\mathrm{y}^{4} \mathrm{P}_{3 / 2}^{\circ}$ & 47154.51 & & 1 & Beynon $(1977)^{e}$ \\
\hline & $\mathrm{y}^{4} \mathrm{P}_{1 / 2}^{\circ}$ & 47299.29 & $-34(2)$ & -8 & Beynon $(1977)^{e}$ \\
\hline \multirow[t]{5}{*}{$3 d^{5} 4 s\left({ }^{7} S\right) 4 d$} & $\mathrm{e}^{6} \mathrm{D}_{9 / 2}$ & 47207.28 & & 15.5 & Luc \& Gerstenkorn $(1972)^{g}$ \\
\hline & $\mathrm{e}^{6} \mathrm{D}_{7 / 2}$ & 47212.06 & & 15.8 & Luc \& Gerstenkorn $(1972)^{g}$ \\
\hline & $\mathrm{e}^{6} \mathrm{D}_{5 / 2}$ & 47215.61 & & 17.6 & Luc \& Gerstenkorn $(1972)^{g}$ \\
\hline & $\mathrm{e}^{6} \mathrm{D}_{3 / 2}$ & 47218.15 & & 22.8 & Luc \& Gerstenkorn $(1972)^{g}$ \\
\hline & $\mathrm{e}^{6} \mathrm{D}_{1 / 2}$ & 47219.64 & & 61.6 & Luc \& Gerstenkorn $(1972)^{g}$ \\
\hline $3 d^{5} 4 s\left({ }^{5} S\right) 5 s$ & $\mathrm{f}^{6} \mathrm{~S}_{5 / 2}$ & 49415.35 & $-20.6(3)$ & -22 & Beynon $(1977)^{e}$ \\
\hline $3 d^{5} 4 s\left({ }^{5} S\right) 5 s$ & $\mathrm{e}^{4} \mathrm{~S}_{3 / 2}$ & 49591.51 & $-50.5(4)$ & -49 & Fisher \& Peck $(1939)^{h}$ \\
\hline $3 d^{5} 4 s\left({ }^{7} S\right) 6 s$ & g ${ }^{6} S_{5 / 2}$ & 50904.68 & $23.2(5)$ & & \\
\hline
\end{tabular}

${ }^{a}$ Spin-exchange optical pumping experiment.

${ }^{b}$ Laser-rf double resonance.

${ }^{c}$ Level crossing.

${ }^{d}$ Fitting of solar profiles.

${ }^{e}$ Reanalysis of old data. These results are quoted only when more accurate data are not available.

Calculated values are given between parentheses.

${ }^{f}$ Laser-atomic beam spectroscopy.

${ }^{g}$ Fabry-Perot measurements and Fourier transform spectroscopy.

${ }^{h}$ Hollow cathode discharge and Fabry-Perot interferometer.

* The notation and energy level values are taken from Sugar \& Corliss (1985). 
for the levels considered and it is expected that they will help astrophysicists in refining the determination of the chemical composition of some stars.

Acknowledgements. We would like to thank the Belgian FNRS, IISN and FRIA for financial support. P.-H. Lefèbvre is FRIA fellow and E. Biémont is Research Director of the FNRS. NSO/Kitt Peak FTS data used here were produced by NSF/NOAO. An initial version of the computer code used was provided to us by M. Godefroid (Brussels).

\section{References}

Aboussaid, A., Carleer, M., Hurtmans, D., Biémont, E., \& Godefroid, M. R. 1996, Phys. Scr., 53, 28

Andriessen, J., Ray, S. N., Lee, T., Das, T. P., \& Ikenberry, D. 1976, Phys. Rev. A, 13, 1669

Bell, J. R. 1972, Introductory Fourier Transform Spectroscopy (New York: Academic)

Beynon, T. G. R. 1977, A\&A, 61, 853

Beynon, T. G. R. 1978a, A\&A, 64, 145

Beynon, T. G. R. 1978b, A\&A, 64, 299

Booth, A. J., \& Blackwell, D. E. 1983, MNRAS, 204, 777

Booth, A. J., Shallis, M. J., \& Wells, M. 1983, MNRAS, 205, 191

Casimir, H. B. G. 1963, On the Interaction Between Atomic Nuclei and Electrons (San Francisco: Freeman)

Cowan, R. D. 1981, The Theory of Atomic Structure and Spectra (Berkeley: University of California Press), 646

Davis, S. J., Wright, J. J., \& Balling, L. C. 1971, Phys. Rev. A, 3, 1220
Dembczyński, J., Ertmer, W., Johann, U., Penselin, S., \& Stinner, P. 1979, Z. Phys. A, 291, 207

Fisher, R. A., \& Peck, E. R. 1939, Phys. Rev., 55, 270

Handrich, E., Steudel, A., \& Walther, H. 1969, Phys. Lett., 29A, 486

Heide, K. V. D. 1968, ZAp, 69, 220

Kronfeldt, H.-D., Kropp, J.-R., Subaric, A., \& Winkler, R. 1985, Z. Phys. A, 322, 349

Kurucz, R. L. 1993, Phys. Scr., T47, 110

Lefèbvre, P.-H., Garnir, H.-P., \& Biémont, E. 2002, Phys. Scr., 66, 363

Luc, P., \& Gerstenkorn, S. 1972, A\&A, 18, 209

Melendez, J. 1999, MNRAS, 307, 197

Murakawa, K., \& Kamei, T. 1953, Phys. Rev., 92, 325

Murakawa, K. 1955, J. Phys. Soc. Japan, 10, 336

Nissen, P. E., Chen, Y. Q., Schuster, W. J., \& Zhao, G. 2000, A\&A, 353,722

Olsson, G., \& Rosén, A. 1982, Phys. Rev. A, 25, 658

Palmeri, P., Biémont, E., Aboussaid, A., \& Godefroid, M. 1995, J. Phys. B: At. Mol. Phys., 28, 3741

Palmeri, P., Biémont, E., Quinet, P., et al. 1997, Phys. Scr., 55, 586

Prochaska, J. X., \& McWilliam, A. 2000, ApJ, 537, L57

Steffen, M. 1985, A\&AS, 59, 403

Sugar, J., \& Corliss, J. 1985, J. Phys. Chem. Ref. Data, 14, Suppl 2., 338

Swensson, J. W. 1966, Ark. Fys., 32, 463

Wali, M., \& Sharma, P. N. 1934, Phil. Mag., 18, 1144

Walther, H. 1962, Z. Phys., 170, 507

White, H. E., \& Ritschl, R. 1930a, Phys. Rev., 35, 208

White, H. E., \& Ritschl, R. 1930b, Phys. Rev., 35, 1146 